\title{
Synthesis of New Rhodanine, Thiazole, Quinazolin-4-One, Imidazolone and Pyranothiazole Derivatives Incorporating Antipyrine Moiety as Antimicrobial Agents
}

\author{
Mahmoud Mohamed Abdelall ${ }^{1,2}$ \\ ${ }^{1}$ Chemistry Department, Faculty of Science, Al-Azhar University, Nasr City, Cairo, Egypt \\ ${ }^{2}$ Chemistry Department, Faculty of Science and Art, Al-Baha University, Al-Baha, Saudi Arabia \\ Email address: \\ abdelall_sci@yahoo.com
}

\section{To cite this article:}

Mahmoud Mohamed Abdelall. Synthesis of New Rhodanine, Thiazole, Quinazolin-4-One, Imidazolone and Pyranothiazole Derivatives Incorporating Antipyrine Moiety as Antimicrobial Agents. American Journal of Heterocyclic Chemistry. Vol. 3, No. 2, 2017 , pp. 8-18. doi: 10.11648/j.ajhc.20170302.11

Received: April 15, 2017; Accepted: May 10, 2017; Published: June 5, 2017

\begin{abstract}
A new series of heterocycles incorporating antipyrine moiety were synthesized via reactions of 4-aminoantipyrine 1 with carbon disulphide then alkylation and /or chloroacetic acid to produce dithiocarbamate and rhodanine 3,4 and 9 . Multi-component reaction (MCR) of 4-aminoantipyrine with carbon disulfide and DMAD afforded rhodanine 7 . The interaction of 9 with diazonium salt, DMF-DMA, nitrous acid and aromatic aldehydes to give thiazolidinone derivatives 10, 11, 13 and 14, respectively. Pyranothiazole 16 was prepared from reaction of 9 with cinamonitrile 15 . Thiazolidinone 18 and thiazole 19 derivatives were obtained from reactions of 9 with phenyl isothiocyanate in the presence of $\mathrm{KOH}$ then halogenated the pot salt with ethyl bromoacetate and chloroacetone, respectively. Thiourea derivative 20 was prepared from reaction of methyl dithiocarbamate 3 with 4-aminoantipyrine. pyrimidine derivatives 21 and 22 were obtained from cyclocondensation of 3 with dimer of ethyl cyanoacetate and malononitrile. Pyrazolotriazine 24 was prepared from interaction of 3 with hydrazine hydrate. copounds 26 and 28 were obtained via reaction of 3 with glycine and anthranilic acid. Compounds 30,32 and 34 were prepared via reaction of thiourea 20 with EAA, ECA and DMAD. Reaction of diazonium chloride of 1 with NaN3 afforded azide 36 which rearranged to triazine 39 . The structures of the newly synthesized compounds were elucidated via elemental analysis and spectral data. The synthesized products were evaluated for their antimicrobial and anti-fungal activity.
\end{abstract}

Keywords: Antipyrine, Rhodanine, Thiazole, Pyranothiazole, Antimicrobial Activity

\section{Introduction}

Antipyrine (1-phenyl-2,3-dimethylpyrazol-5-one) and its derivatives have been reported to possess varied biological activities such as analgesic [1,2], anti-inflammatory [3], antimicrobial [4-6], and anticancer activity [7], antiviral [8, 9], clinical [10], and pharmacological [11, 12] activities. Furthermore, rhodanine derivatives possess anticonvulsant, antibacterial, antiviral, and antidiabetic activities [13 - 15] Some of rhodanine-based derivatives act as hepatitis $\mathrm{C}$ virus (HCV) protease inhibitor [16]. The present work was demonstrated a strategy for the synthesis of novel rhodanine, thiazole, quinazolin-4-one, imidazolone and pyranothiazole bearing antipyrine moiety and investigated their antimicrobial potential activities.

\section{Material and Methods}

Melting points were determined using an Electro thermal Gallenkamp apparatus and are reported uncorrected. IR spectra were recorded in $\mathrm{KBr}$ using PyUnicam SP-1000 Spectrometer. ${ }^{1} \mathrm{H}-\mathrm{NMR}$ spectra were recorded using $\mathrm{CDCl}_{3}$ and DMSO-d6 solutions using a Varian Em-400 MHz Spectrometer and chemical shifts are reported in ppm. relative to that of TMS, which was used as an internal standard (measured at faculty of pharmacy - Cairo University). Mass spectra were recorded using AEI MS 30 mass spectrometer operating at $70 \mathrm{eV}$. Elemental analyses were carried out by the Micro analytical Center of Cairo University, Giza, Egypt. Mass spectra were carried out at the Regional Center for Mycology and Biotechnology at Al-Azhar University, Cairo, 
Egypt, Antimicrobial activities measured at faculty of agriculture, Al-Azhar University.

\subsection{General Procedure for Synthesis of Compounds 3,4,7 and 8 (Figure 1)}

\subsubsection{General Procedure for Synthesis of Alkyl Dithiocarbamate 3, 4 and Thiazole 8}

A solution of 4 -aminoantipyrine $(0.01 \mathrm{~mol})$ in ethanol $(20$ $\mathrm{mL}$ ) was stirred vigorously. To this was added finally powdered potassium hydroxide $(0.01 \mathrm{~mol})$ and carbon disulphide $(0.01 \mathrm{~mol})$ during $30 \mathrm{~min}$ with stirring. Methyl iodide and /benzyl chloride /chloroacetone $(0.01 \mathrm{~mol})$ was then added gradually keeping the reaction mixture in freezing mixture with stirring, and the stirring was continued for further $2 \mathrm{~h}$. The reaction mixture was then poured into ice water. The solid obtained was filtered, washed with water, dried, and recrystallized from ethanol.

(i) Methyl(1,5-dimethyl-3-oxo-2-phenyl-2,3-dihydro-1H-p yrazol-4-yl)carbamodithioate (3)

Yield $84 \%$; white crystals ; mp $160-162^{\circ} \mathrm{C}$; IR (KBr): $\left(v / \mathrm{cm}^{-1}\right)=3125(\mathrm{NH}), 3063(\mathrm{CH}$ aromatic $), 2920(\mathrm{CH}$ aliphatic), $1644(\mathrm{CO}), 1283(\mathrm{C}=\mathrm{S}) ;{ }^{1} \mathrm{HNMR}$ spectrum, $\delta$, ppm: $2.18\left(\mathrm{~s}, 3 \mathrm{H}, \mathrm{CCH}_{3}\right), 2.60\left(\mathrm{~s}, 3 \mathrm{H}, \mathrm{SCH}_{3}\right) 3.26\left(\mathrm{~s}, 3 \mathrm{H}, \mathrm{NCH}_{3}\right), 7.29$ 7.55 (m, 5H, Ar-H), 10.96 (s, 1H, NH); Anal. Calcd. For $\mathrm{C}_{13} \mathrm{H}_{15} \mathrm{~N}_{3} \mathrm{OS}_{2}$ (293): Calcd.: C, 53.24; H, 5.11; N, $14.33 \%$; Found: C, 53.21; H, 5.07; N, 14.29\%.

\section{(ii) Benzyl(1,5-dimethyl-3-oxo-2-phenyl-2,3-dihydro-1H- pyrazol-4-yl)carbamodithioate (4)}

Yield $86 \%$; white crystals ; mp $175-177^{\circ} \mathrm{C}$; IR (KBr): $\left(\mathrm{v} / \mathrm{cm}^{-1}\right)=3132(\mathrm{NH}), 3051(\mathrm{CH}$ aromatic $), 2926(\mathrm{CH}$ aliphatic $)$, $1640(\mathrm{CO}), 1283\left(\mathrm{C}=\mathrm{S}, ;{ }^{1} \mathrm{HNMR}\right.$ spectrum, $\delta$, ppm: 2.28 ( s, 3H, $\left.\mathrm{CCH}_{3}\right), 3.19\left(\mathrm{~s}, 3 \mathrm{H}, \mathrm{NCH}_{3}\right), 4.53\left(\mathrm{~s}, 2 \mathrm{H}, \mathrm{SCH}_{2}\right), 7.25-7.48(\mathrm{~m}$, $10 \mathrm{H}, \mathrm{Ar}-\mathrm{H}), 10.70(\mathrm{~s}, 1 \mathrm{H}, \mathrm{NH})$; Anal. Calcd. For $\mathrm{C}_{19} \mathrm{H}_{19} \mathrm{~N}_{3} \mathrm{OS}_{2}$ (369): Calcd.: C, 61.78; H, 5.14; N, $11.38 \%$; Found: C, 61.73; H, $5.09 ; \mathrm{N}, 11.32 \%$.

\section{(iii) 1,5-dimethyl-4-(4-methyl-2-thioxothiazol-3(2H)-}

\section{yl)-2-phenyl-1,2-dihydro-3H-pyrazol-3-one (8)}

Yield $84 \%$; pale yellow crystals ; mp $250-252^{\circ} \mathrm{C}$; IR $(\mathrm{KBr})$ $\left(\mathrm{v} / \mathrm{cm}^{-1}\right)=3096(\mathrm{CH}$ aromatic), 2923(CH aliphatic), 1669 (CO), 1263(C=S); ${ }^{1} \mathrm{HNMR}$ spectrum, $\delta$, ppm: $2.09\left(\mathrm{~s}, 3 \mathrm{H}, \mathrm{CH}_{3}\right.$ of thiazole) $2.19\left(\mathrm{~s}, 3 \mathrm{H}, \mathrm{CCH}_{3}\right), 3.31 \quad\left(\mathrm{~s}, 3 \mathrm{H}, \mathrm{NCH}_{3}\right)$, 6.26(s,1H,C-H5 of thiazole), 7.25-7.64 (m, 5H, Ar-H); Anal. Calcd. For $\mathrm{C}_{15} \mathrm{H}_{15} \mathrm{~N}_{3} \mathrm{OS}_{2}$ (317): Calcd.: C, 56.76; H, 4.76; N, $13.24 \%$; Found: C, 56.72; H, 4.775; N, $13.21 \%$.

\subsubsection{Methy l-2-(3-(1, 5-dimethyl-3-oxo-2-phenyl-2, 3- dihydro-1H-pyrazol-4-yl)-4-oxo-2-thioxothiazo-lidin- 5-ylidene) Acetate (7)}

A mixture of 4-aminoantipyrine $(0.01 \mathrm{~mol})$, carbon disulphide $(0.01 \mathrm{~mol})$ and DMAD $(0.01 \mathrm{~mol})$ was stirred in ethanol $(20 \mathrm{~mL})$ for $4 \mathrm{hrs}$. A red solid was obtained and filtered. The solid was recrystallized from ethanol to give 7 as red crystal (Yield $86 \%$ ) mp $83-85^{\circ} \mathrm{C}$; IR (KBr): $\left(\mathrm{v} / \mathrm{cm}^{-1}\right.$ ) $=3001$ (CH aromatic), 2951 (CH aliphatic), 1741, 1671 (CO),1231(C=S); ${ }^{1} \mathrm{HNMR}$ spectrum, $\delta$, ppm: 2.34 ( s, 3H,
$\left.\mathrm{CCH}_{3}\right), 3.41\left(\mathrm{~s}, 3 \mathrm{H}, \mathrm{NCH}_{3}\right), 3.74\left(\mathrm{~s}, 3 \mathrm{H}, \mathrm{COOCH}_{3}\right), 7.26-7.48$ (m, $6 \mathrm{H}$, Ar-H and $\mathrm{C}=\mathrm{CH})$; Anal. Calcd. For $\mathrm{C}_{17} \mathrm{H}_{15} \mathrm{~N}_{3} \mathrm{O}_{4} \mathrm{~S}_{2}$ (389): Calcd.: C, 52.44; H, 3.85; N, $10.79 \%$; Found: C, 52.41; H, 3.82; N, $10.75 \%$.

\subsection{General Procedure for Synthesis of Compounds 9-16 (Figure 2)}

\subsection{1. (1,5-dimethyl-3-oxo-2-phenyl-2,3-dihydro-1H-pyr- azol-4-yl)-2-thioxothiazolidin-4-one (9)}

A solution of 4-aminoantipyrine $(0.01 \mathrm{~mol})$ in ethanol $(20$ $\mathrm{mL}$ ) was stirred vigorously. To this was added finally powdered potassium hydroxide $(0.01 \mathrm{~mol})$ and carbon disulphide $(0.01 \mathrm{~mol})$ during $30 \mathrm{~min}$ with stirring. To this was then added a solution made up of $1 \mathrm{~g}$ chloroacetic acid, $20 \mathrm{cc}$ of water and enough sodium carbonate to give a neutral solution. The whole was stirred for a further 1 hour. The mixture was then acidified with conc. Hydrochloric acid and heated one hour on a steam bath. The product then separated on chilling and was filtered and dried. recrystallized from ethanol to give 9 as pale yellow crystals (Yield $71 \%$ ) ; mp 114-116 ${ }^{\circ}$; IR $(\mathrm{KBr}):\left(\mathrm{v} / \mathrm{cm}^{-1}\right)=3058(\mathrm{CH}$ aromatic $), 2979$ (CH aliphatic) 1743, $1664(\mathrm{CO}), 1240(\mathrm{C}=\mathrm{S}) ;{ }^{1} \mathrm{HNMR}$ spectrum, $\delta$, ppm: $2.20\left(\mathrm{~s}, 3 \mathrm{H}, \mathrm{CCH}_{3}\right), 3.30\left(\mathrm{~s}, 3 \mathrm{H}, \mathrm{NCH}_{3}\right)$, 4.22(s, $\left.2 \mathrm{H}, \mathrm{CH}_{2}\right), 7.02-7.53$ (m, 5H, Ar-H); Anal. Calcd. For $\mathrm{C}_{14} \mathrm{H}_{13} \mathrm{~N}_{3} \mathrm{O}_{2} \mathrm{~S}_{2}$ (319): Calcd.: C, 52.66; H, 4.07; N, $13.16 \%$; Found: C, 52.63; H, 4.01; N, 13.12\%.

\subsubsection{3-(1,5-dimethyl-3-oxo-2-phenyl-2,3-dihydro-1H- pyrazol-4-yl)-5-((1,5-dimethyl-3-oxo-2-phenyl-2,3-dih ydro-1H-pyrazol-4-yl)diazenyl)-2-thioxothiaz-olidin-4 -one (10)}

To a cold solution of $9(0.01 \mathrm{~mol})$ in ethanol $(30 \mathrm{~mL})$ was added antipyrene diazonium chloride $(0.01 \mathrm{~mol})$ [prepared by diazotization of 4-aminoantipyrine $1 \quad(0.012 \mathrm{~mol})$ in concentrated $\mathrm{HCl}(6 \mathrm{ml})$ with sodium nitrite $(0.97 \mathrm{~g}$ in $5 \mathrm{ml}$ $\mathrm{H}_{2} \mathrm{O}$ ) at $0^{\circ} \mathrm{C}$ ] portion wise over $30 \mathrm{~min}$. with constant stirring. After complete addition, the reaction mixture was stirred for a further $3 \mathrm{~h}$ at $0^{\circ} \mathrm{C}$, the solid product was obtained filtered off, washed with water, dried and recrystallized from ethnol to give 10 as red crystals (Yield $76 \%$ ); mp $101-103^{\circ} \mathrm{C}$; IR (KBr): $\left(\mathrm{v} / \mathrm{cm}^{-1}\right)=3060(\mathrm{CH}$ aromatic), 2979 (CH aliphatic) 1744, 1656 (CO), 1239 (C=S); MS (m/z): $533(\mathrm{M}+, 56 \%)$; Anal. Calcd. For $\mathrm{C}_{25} \mathrm{H}_{23} \mathrm{~N}_{7} \mathrm{O}_{3} \mathrm{~S}_{2}$ (533): Calcd.: C, 56.28; H, 4.31; N, 18.38 \%; Found: C, 56.23; H, 4.28; N, $18.34 \%$.

\subsubsection{3-(1,5-dimethyl-3-oxo-2-phenyl-2,3-dihydro-1H- pyrazol-4-yl)-5-(dimethylamino)methylene)-2-thioxot hiazolidin-4-one (11)}

A solution of rhodanine $9 \quad(0.01 \quad \mathrm{~mol})$ and dimethylformamide dimethylacetal $(0.01 \mathrm{~mol})$ in benzene $(30$ $\mathrm{mL}$ ) was refluxed for $3 \mathrm{~h}$. The removal of the solvent under reduced pressure yielded the crude product which was crystallized from ethanol to give 11 as purple crystals(Yield $69 \%) ; \mathrm{mp} 209-211^{\circ} \mathrm{C}$; IR $(\mathrm{KBr}):\left(\mathrm{v} / \mathrm{cm}^{-1}\right)=3048(\mathrm{CH}$ aromatic), 2918 (CH aliphatic), 1683 (CO), 1277 (C=S); ${ }^{1} \mathrm{HNMR}$ spectrum, $\delta$, ppm: $2.17 \quad\left(\mathrm{~s}, 3 \mathrm{H}, \mathrm{CCH}_{3}\right), \quad 3.20$ $\left(\mathrm{s}, 6 \mathrm{H}, \mathrm{N}\left(\mathrm{CH}_{3}\right)_{2}\right), .48\left(\mathrm{~s}, 3 \mathrm{H}, \mathrm{NCH}_{3}\right), 7.29-7.58(\mathrm{~m}, 6 \mathrm{H}, \mathrm{Ar}-\mathrm{H}$ 
and $\mathrm{C}=\mathrm{CH}$ ); Anal. Calcd. For $\mathrm{C}_{17} \mathrm{H}_{18} \mathrm{~N}_{4} \mathrm{O}_{2} \mathrm{~S}_{2}$ (374): Calcd.: $\mathrm{C}$, 54.54; H, 4.81; N, $19.97 \%$; Found: C, 54.51; H, 4.78; N, $19.95 \%$.

\subsubsection{3-(1,5-dimethyl-3-oxo-2-phenyl-2,3-dihydro-1H-} pyrazol-4-yl)-5-nitroso-2-thioxothiazolidin-4-one (12)

A solution of $9(0.01 \mathrm{~mol})$ in acetic acid $(20 \mathrm{~mL})$ was treated with aqueous solution of sodium nitrite while stirring for 1 hour. The yellow product which precipitated was filtered and washed with water then recrystallized from acetic acid to give 12 as yellow crystals (Yield $74 \%$ ); mp $247-249^{\circ} \mathrm{C}$; IR (KBr): $\left(\mathrm{v} / \mathrm{cm}^{-1}\right)=3068(\mathrm{CH}$ aromatic), $2975(\mathrm{CH}$ aliphatic $), 1747$ (CO), $1577(\mathrm{NO}), 1227(\mathrm{C}=\mathrm{S}) ;{ }^{1} \mathrm{HNMR}$ spectrum, $\delta$, ppm: $2.23\left(\mathrm{~s}, 3 \mathrm{H}, \mathrm{CCH}_{3}\right), 3.27\left(\mathrm{~s}, 3 \mathrm{H}, \mathrm{NCH}_{3}\right), 7.36-7.57(\mathrm{~m}, 5 \mathrm{H}$, Ar-H ), $14.00(\mathrm{~s}, 1 \mathrm{H}, \mathrm{OH})$; Anal. Calcd. For $\mathrm{C}_{14} \mathrm{H}_{12} \mathrm{~N}_{4} \mathrm{O}_{3} \mathrm{~S}_{2}$ (348) Calcd.: C, 48.27; H, 3.44; N, $16.09 \%$; Found: C,48.24; H, $3.41 ; \mathrm{N}, 16.05 \%$.

\subsubsection{General Procedures for Synthesis of Arylidine 14a,b}

To a solution of $9(0.01 \mathrm{~mol})$ in ethanol $(30 \mathrm{~mL})$, appropriate aldehyde $(0.01 \mathrm{~mol})$, and piperidine $(0.5 \mathrm{~mL})$ were added. The mixture was refluxed for 3 hours, after cooling, the resulting precipitate was filtered off, dried and recrystallized from ethanol.

(i) 3-(1,5-dimethyl-3-oxo-2-phenyl-2,3-dihydro-1Hpyrazol-4-yl)-5-(4-methoxybenzylidene)-2-thioxothiazo lidin-4-one (14a)

Yield 73\%; yellow crystals; mp $237-239^{\circ} \mathrm{C}$; IR (KBr): $\left(\mathrm{v} / \mathrm{cm}^{-1}\right)=3068(\mathrm{CH}$ aromatic $), 2969(\mathrm{CH}$ aliphatic) 1720,1679 (CO), $1265(\mathrm{C}=\mathrm{S})$; ${ }^{1} \mathrm{HNMR}$ spectrum, $\delta$, ppm: $2.21(\mathrm{~s}, 3 \mathrm{H}$, $\left.\mathrm{CCH}_{3}\right), 3.27\left(\mathrm{~s}, 3 \mathrm{H}, \mathrm{NCH}_{3}\right), 3.86\left(\mathrm{~s}, 3 \mathrm{H}, \mathrm{OCH}_{3}\right) 7.15-7.69(\mathrm{~m}, 9 \mathrm{H}$ Ar-H), $7.86(\mathrm{~s}, 1 \mathrm{H}, \mathrm{C}=\mathrm{CH})$; Anal. Calcd. For $\mathrm{C}_{22} \mathrm{H}_{19} \mathrm{~N}_{3} \mathrm{O}_{3} \mathrm{~S}_{2}$ (437): Calcd.: C, 60.41; H, 4.34; N, 9.61\%; Found: C, 60.38; $\mathrm{H}, 4.31 ; \mathrm{N}, 9.58 \%$.

(ii) 3-(1,5-dimethyl-3-oxo-2-phenyl-2,3-dihydro-1Hpyrazol-4-yl)-5-(4-ethoxy-3-hydroxybenzylidene)-2-thi oxothiazolidin-4-one (14b)

Yield 78\%; yellow crystals; mp $228-230^{\circ} \mathrm{C}$; IR $(\mathrm{KBr}):\left(\mathrm{v} / \mathrm{cm}^{-1}\right)=3444(\mathrm{OH}), 3060(\mathrm{CH}$ aromatic $), 2972(\mathrm{CH}$ aliphatic), 1714,1656(CO), 1223(C=S); ${ }^{1} \mathrm{HNMR}$ spectrum, $\delta$, ppm: $\quad 1.37\left(\mathrm{t}, 3 \mathrm{H}, \mathrm{OCH}_{2} \mathrm{CH}_{3}\right) 2.20 \quad\left(\mathrm{~s}, \quad 3 \mathrm{H}, \quad \mathrm{CCH}_{3}\right), \quad 3.33$ $\left(\mathrm{s}, 3 \mathrm{H}, \mathrm{NCH}_{3}\right), 4.25\left(\mathrm{q}, 2 \mathrm{H}, \mathrm{OCH}_{2} \mathrm{CH}_{3}\right), 4.93(\mathrm{br}, 1 \mathrm{H}, \mathrm{OH}), 6.75-$ $7.52(\mathrm{~m}, 9 \mathrm{H}, \mathrm{Ar}-\mathrm{H}), 7.86(\mathrm{~s}, 1 \mathrm{H}, \mathrm{C}=\mathrm{CH})$; Anal. Calcd. For $\mathrm{C}_{23} \mathrm{H}_{21} \mathrm{~N}_{3} \mathrm{O}_{4} \mathrm{~S}_{2}$ (467): Calcd.: C, 59.10; H, 4.49; N, 8.99\%; Found: C, 59.06; H, 4.45; N, 8.96\%.

\subsubsection{General Procedures Synthesis of Pyrano [2,3-d]- Thiazole 16a,b}

To a solution of $9(0.01 \mathrm{~mol})$ and the appropriate cinnamonitrile $(0.01 \mathrm{~mol})$ in ethanol $(30 \mathrm{~mL})$ containing piperidine $(0.5 \mathrm{~mL})$. The reaction mixture, was heated under reflux for 3 hours, the obtained product on heating, was collected by filtration. Then recrystallized from the proper solvent. (i) 5-amino-3-(1,5-dimethyl-3-oxo-2-phenyl-2,3-dihydro -1H-pyrazol-4-yl)-7-(4-methoxy-phenyl)-2-thioxo-3,7dihydro-2H-pyrano[2,3-d]thiazole-6-carbonitrile (16a) $\mathrm{AcOH}$ (Yield 63\%); pale yellow crystals; mp $247-249^{\circ} \mathrm{C}$; IR $(\mathrm{KBr}):\left(\mathrm{v} / \mathrm{cm}^{-1}\right)=3385,3330\left(\mathrm{NH}_{2}\right), 3061(\mathrm{CH}$ aromatic $)$, 2969 (CH aliphatic), 2200 (CN),1713 (CO), 1263(C=S); ${ }^{1} \mathrm{HNMR}$ spectrum, $\delta$, ppm: 2.21 ( $\left.\mathrm{s}, 3 \mathrm{H}, \mathrm{CCH}_{3}\right), 3.31$ $\left(\mathrm{s}, 3 \mathrm{H}, \mathrm{NCH}_{3}\right), 3.83\left(\mathrm{~s}, 3 \mathrm{H}, \mathrm{OCH}_{3}\right), 7.23-7.74(\mathrm{~m}, 9 \mathrm{H}, \mathrm{Ar}-\mathrm{H})$, $8.56\left(\mathrm{~s}, 1 \mathrm{H}\right.$, pyran-H4); Anal. Calcd. For $\mathrm{C}_{25} \mathrm{H}_{21} \mathrm{~N}_{5} \mathrm{O}_{3} \mathrm{~S}_{2}(503)$ : Calcd.: C, 59.63; H, 4.20; N, 13.91\%; Found: C, 59.60; H, $4.15 ; \mathrm{N}, 13.88 \%$.

(ii) 5-amino-3-(1,5-dimethyl-3-oxo-2-phenyl-2,3-dihydro -1H-pyrazol-4-yl)-7-(4-ethoxy-3-hydroxyphenyl)-2-thio xo-3,7-dihydro-2H-pyrano[2,3-d]thiazole-6-carbonitril e (16b)

$\mathrm{AcOH}$ (Yield 77\%); pale yellow crystals; mp 270-272 ${ }^{\circ} \mathrm{C}$; IR $(\mathrm{KBr}):\left(\mathrm{v} / \mathrm{cm}^{-1}\right)=3526(\mathrm{OH}), 3386,3324\left(\mathrm{NH}_{2}\right), 3051(\mathrm{CH}$ aromatic), 2981 ( $\mathrm{CH}$ aliphatic),2193 (CN),1707 (CO), 1274(C=S); ${ }^{1} \mathrm{HNMR}$ spectrum, $\delta$, ppm: $1.35\left(\mathrm{t}, 3 \mathrm{H}, \mathrm{OCH}_{2} \mathrm{CH}_{3}\right)$, $2.51 \quad\left(\mathrm{~s}, \quad 3 \mathrm{H}, \quad \mathrm{CCH}_{3}\right), \quad 3.33\left(\mathrm{~s}, 3 \mathrm{H}, \mathrm{NCH}_{3}\right), 4.09$ $\left(\mathrm{q}, 2 \mathrm{H}, \mathrm{OCH}_{2} \mathrm{CH}_{3}\right), 4.45(\mathrm{~s}, 1 \mathrm{H}, \mathrm{OH}), 6.78-7.76(\mathrm{~m}, 8 \mathrm{H}, \mathrm{Ar}-\mathrm{H})$,

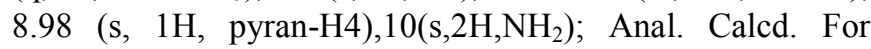
$\mathrm{C}_{26} \mathrm{H}_{23} \mathrm{~N}_{5} \mathrm{O}_{4} \mathrm{~S}_{2}$ (533): Calcd.: C, 58.52; H, 4.34; N, 13.12\%; Found: C, 58.50; H, 4.32; N, 13.09\%.

\subsection{General Procedure for Synthesis of Compounds 18 and 19 (Figure 3)}

To a solution of compound $9(0.01 \mathrm{~mol})$ in dimethylformamide $(20 \mathrm{~mL})$ containing potassium hydroxide $(0.01 \mathrm{~mol})$ phenyl isothiocyanate $(0.01 \mathrm{~mol})$ was added. The reaction mixture was stirred at room temperature overnight. To the reaction mixture either of ethyl $\alpha$ - bromoacetate $(0.01$ mol) or chloroacetone $(0.01 \mathrm{~mol})$ was added and the whole reaction mixture was stirred at room temperature overnight. The solid product, so formed in each case, upon pouring onto ice/water containing hydrochloric acid (till $\mathrm{pH}$ 6) was collected by filtration and crystallized from ethanol.

\subsubsection{3'-(1,5-dimethyl-3-oxo-2-phenyl-2,3-dihydro-1H- pyrazol-4-yl)-3-phenyl-2'-thioxo-2',3'-dihydro- 3H,4'H-[2,5'-bithiazolylidene]-4,4'(5H)-dione (18)}

Yield 71\%; red crystals; mp 294-296 ${ }^{\circ} \mathrm{C}$; IR (KBr): $\left(\mathrm{v} / \mathrm{cm}^{-1}\right)$ $=3057$ (CH aromatic), 2982 (CH aliphatic), 1713,1673 (CO), 1256(C=S); ${ }^{1} \mathrm{HNMR}$ spectrum, $\delta$, ppm: 2.19 ( s, 3H, $\left.\mathrm{CCH}_{3}\right)$, 3.34(s,3H, $\left.\mathrm{NCH}_{3}\right), 3.92\left(\mathrm{~s}, 2 \mathrm{H}, \mathrm{CH}_{2}\right), 7.23-7.59(\mathrm{~m}, 10 \mathrm{H}, \mathrm{Ar}-\mathrm{H})$; Anal. Calcd. For $\mathrm{C}_{23} \mathrm{H}_{18} \mathrm{~N}_{4} \mathrm{O}_{3} \mathrm{~S}_{3}$ (494): Calcd.: C, 55.85; H, 3.67; N, 11.33\%; Found: C, 55.81; H, 3.62; N, 11.31\%.

\subsubsection{3-(1,5-dimethyl-3-oxo-2-phenyl-2,3-dihydro-1H- pyrazol-4-yl)-5-(4-methyl-3-phenylthiazol-2(3H)-ylide ne)-2-thioxothiazolidin-4-one (19)}

Yield 75\%; brwon crystals; mp $364-366^{\circ} \mathrm{C}$; IR $(\mathrm{KBr})$ : $\left(v / \mathrm{cm}^{-1}\right)=3074(\mathrm{CH}$ aromatic $), 2918(\mathrm{CH}$ aliphatic $), 1658$ (CO), 1231 $(\mathrm{C}=\mathrm{S})$; ${ }^{1} \mathrm{HNMR}$ spectrum, $\delta$, ppm: $1.90(\mathrm{~s}, 3 \mathrm{H}$, $\left.\mathrm{CH}_{3}\right), 2.21\left(\mathrm{~s}, 3 \mathrm{H}, \mathrm{CCH}_{3}\right), 3.31\left(\mathrm{~s}, 3 \mathrm{H}, \mathrm{NCH}_{3}\right), 6.95(\mathrm{~s}, \mathrm{H}$,

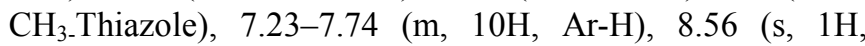
pyran-H4); Anal. Calcd. For $\mathrm{C}_{24} \mathrm{H}_{20} \mathrm{~N}_{4} \mathrm{O}_{2} \mathrm{~S}_{3}$ (492): Calcd.: C, 
58.52; H, 4.09; N, 11.37\%; Found: C, 58.48; H, 4.05; N, $11.33 \%$.

\subsection{General Procedure for Synthesis of Compounds 20-29(Figure 4).}

\subsubsection{1,3-bis(1,5-dimethyl-3-oxo-2-phenyl-2,3-dihydro- 1H-pyrazol-4-yl)thiourea (20)}

To a solution of $3(0.01 \mathrm{~mol})$, in ethanol $(20 \mathrm{ml}$.) 4-aminoantipyrine $(0.01 \mathrm{~mol})$ and TEA $(0.5 \mathrm{~mL})$ were added. The reaction mixture was heated under reflux for 2 hours then left to cool and the formed solid product was collected by filtration and crystallized from acetic acid to give 20, Yield $79 \%$; brown crystals; mp $253-255^{\circ} \mathrm{C}$; IR $(\mathrm{KBr}):\left(\mathrm{v} / \mathrm{cm}^{-1}\right)=$ $3253(\mathrm{NH}), 3051$ ( $\mathrm{CH}$ aromatic), 2936 (CH aliphatic), 1661 (CO), 1248(C=S); MS (m/z): $448(\mathrm{M}+, 0.72 \%)$; Anal. Calcd. For $\mathrm{C}_{23} \mathrm{H}_{24} \mathrm{~N}_{6} \mathrm{O}_{2} \mathrm{~S}$ (448): Calcd.: C, 61.60; H, 5.35; N, 18.75\%; Found: C, 61.56; H, 5.32; N, 18.73\%.

\subsubsection{Reaction of 3 with Dimer of Ethyl Cyanoacetate and Malononitrile}

A mixture of $3(0.01 \mathrm{~mol})$ and dimer of ethyl cyanoacetate and /dimer of malononitrile $(0.01 \mathrm{~mol})$ in ethanol $(30 \mathrm{ml})$ in the presence of TEA $(0.5 \mathrm{~mL})$ was refluxed for 3 hours. The obtained product on heating was collected by filtration and recrystallized from the proper solvent.

(i) (1,5-dimethyl-3-oxo-2-phenyl-2,3-dihydro-1H-pyrazol4-yl)-6-((ethylperoxy)methyl)-4-oxo-2-thioxo-1,2,3,4tetrahydropyrimidine-5-carbonitrile (21)

EtOH; Yield 74\%; white crystals; mp $170-172^{\circ} \mathrm{C}$; IR (KBr): $\left(v / \mathrm{cm}^{-1}\right)=3144(\mathrm{NH}), 3063(\mathrm{CH}$ aromatic $), 2985(\mathrm{CH}$ aliphatic), $2190(\mathrm{CN}), 1703,1650(\mathrm{CO}), 1267(\mathrm{C}=\mathrm{S}) ;{ }^{1} \mathrm{HNMR}$ spectrum, $\delta$, ppm: 1.31(t,3H, $\left.\mathrm{OCH}_{2} \mathrm{CH}_{3}\right) 2.37\left(\mathrm{~s}, 3 \mathrm{H}, \mathrm{CCH}_{3}\right)$, $3.35\left(\mathrm{~s}, 3 \mathrm{H}, \mathrm{NCH}_{3}\right), 4.20\left(\mathrm{q}, 2 \mathrm{H}, \mathrm{OCH}_{2} \mathrm{CH}_{3}\right), 4.63\left(\mathrm{~s}, 2 \mathrm{H}, \mathrm{CH}_{2}\right)$, 7.29-7.55 (m, 5H, Ar-H),11.20(s,1H,NH); Anal. Calcd. For $\mathrm{C}_{20} \mathrm{H}_{19} \mathrm{~N}_{5} \mathrm{O}_{4} \mathrm{~S}$ (425): Calcd.: C, 56.47; H, 4.47; N, 16.47\%; Found: C, 56.43; H, 4.42; N, 16.45\%.

(ii) 6-(cyanomethyl)-3-(1,5-dimethyl-3-oxo-2-phenyl-2,3-d ihydro-1H-pyrazol-4-yl)-4-imino-2-thioxo-1,2,3,4-tetra hydropyrimidine-5-carbonitrile (22)

$\mathrm{AcOH}$; Yield $82 \%$; white crystals; mp $277-279^{\circ} \mathrm{C}$; IR $(\mathrm{KBr}):\left(\mathrm{v} / \mathrm{cm}^{-1}\right)=3321,3172(2 \mathrm{NH}), 3052(\mathrm{CH}$ aromatic $)$, 2937 (CH aliphatic), $2205(\mathrm{CN}), 1647$ (CO), 1259(C=S); MS $(\mathrm{m} / \mathrm{z}): 448(\mathrm{M}+, 0.72 \%)$; Anal. Calcd. For $\mathrm{C}_{18} \mathrm{H}_{15} \mathrm{~N}_{7} \mathrm{OS}$ (377): Calcd.: C, 57.29; H, 3.97; N, 25.99\%; Found: C, 57.26; H, $3.95 ;$ N, $25.96 \%$.

\subsubsection{Synthesis of Compounds 24, 26 and 28}

A mixture of $3(0.01 \mathrm{~mol})$, and hydrazine hydrate and/or methyl glycinate hydrochloride and/or anthranilic acid $(0.01$ $\mathrm{mol})$ in EtOH $(30 \mathrm{ml})$ and TEA $(0.5 \mathrm{ml}$.). The reaction mixture was heated under reflux for 3 hours or until methanthiol ceased then left to cool and the formed solid product was collected by filtration and crystallized from the proper solvent. (i) 2,3-dimethyl-1-phenyl-1,2,4,6-tetrahydro-5H-pyrazoloI 4,3-e][1,2,4]triazine-5-thione (24)

EtOH; Yield 62\%; white crystals; mp $140-142^{\circ} \mathrm{C}$; IR (KBr): $\left(v / \mathrm{cm}^{-1}\right)=3322(\mathrm{NH}), 3017(\mathrm{CH}$ aromatic $), 2949(\mathrm{CH}$ aliphatic), MS (m/z): 259 (M+, 0.96\%); Anal. Calcd. For $\mathrm{C}_{12} \mathrm{H}_{13} \mathrm{~N}_{5} \mathrm{~S}$ (259): Calcd.: C, 55.59; H, 5.01; N, 27.02\%; Found: C, 55.56; H, 5.03; N, 27.00\%.

\section{(ii) 1,5-dimethyl-4-(5-oxo-2-thioxoimidazolidin-1-yl)-} 2-phenyl-1,2-dihydro-3H-pyrazol-3-one (26)

EtOH; Yield 69\%; brown crystals; mp $245-247^{\circ} \mathrm{C}$; IR $(\mathrm{KBr}):\left(\mathrm{v} / \mathrm{cm}^{-1}\right)=3115(\mathrm{NH}), 3005(\mathrm{CH}$ aromatic $), 2960(\mathrm{CH}$ aliphatic), 1768, $1656(\mathrm{CO}), 1233(\mathrm{C}=\mathrm{S}) ;{ }^{1} \mathrm{HNMR}$ spectrum, $\delta$, ppm: 2.22 ( s, $\left.3 \mathrm{H}, \mathrm{CCH}_{3}\right), 3.20\left(\mathrm{~s}, 3 \mathrm{H}, \mathrm{NCH}_{3}\right), 4.34\left(\mathrm{~s}, 2 \mathrm{H}, \mathrm{CH}_{2}\right)$, 7.35-7.56 (m, 5H, Ar-H), 10.41 (br, 1H,NH); Anal. Calcd. For $\mathrm{C}_{14} \mathrm{H}_{14} \mathrm{~N}_{4} \mathrm{O}_{2} \mathrm{~S}$ (302): Calcd.: C, 56.62; H, 4.63; N, 18.54\%; Found: C, 56.59; H, 4.61; N, 18.51\%.

(iii) 3-(1,5-dimethyl-3-oxo-2-phenyl-2,3-dihydro-1H-pyraz ol-4-yl)-2-thioxo-2,3-dihydroquinazolin- (1H)-one (28)

$\mathrm{AcOH}$; Yield 82\%; white crystals; mp $322-324^{\circ} \mathrm{C}$; IR $(\mathrm{KBr}):\left(\mathrm{v} / \mathrm{cm}^{-1}\right)=3157(\mathrm{NH}), 3066(\mathrm{CH}$ aromatic $), 2921(\mathrm{CH}$ aliphatic), 1698, 1650 (CO), 1263(C=S); MS (m/z): $448(\mathrm{M}+$, 63.88\%).; Anal. Calcd. For $\mathrm{C}_{19} \mathrm{H}_{16} \mathrm{~N}_{4} \mathrm{O}_{2} \mathrm{~S}$ (364): Calcd.: $\mathrm{C}$, 62.63; H, 4.39; N, 15.38\%; Found: C, 62.61; H, 4.35; N, $15.33 \%$.

\subsubsection{3-(1,5-dimethyl-3-oxo-2-phenyl-2,3-dihydro-1H-} pyrazol-4-yl)-2-hydrazinylquinazolin-4(3H)-one (29)

A solution of $28(0.01 \mathrm{~mol})$ in ethanol $(30 \mathrm{ml})$ and hydrazine hydrate $(0.01 \mathrm{~mol})$ was refluxed for 3 hours. The solvent was removed by distillation under reduced pressure and the resulting solution was left to cool. The solid precipitate was collected by filtration and recrystallized from EtOH. Yield 82\%; white crystals; mp $244-246^{\circ} \mathrm{C}$; IR (KBr): $\left(\mathrm{v} / \mathrm{cm}^{-1}\right)=3298,3206\left(\mathrm{NH}_{2}\right), 3066(\mathrm{CH}$ aromatic $), 2941(\mathrm{CH}$ aliphatic), 1675,1640 (CO); MS (m/z): $362(\mathrm{M}+, 1.60 \%)$; Anal. Calcd. For $\mathrm{C}_{19} \mathrm{H}_{18} \mathrm{~N}_{6} \mathrm{O}_{2}$ (362): Calcd.: C, 62.98; H, 4.97; N, 23.20\%; Found: C, 62.95; H, 4.93; N, 23.17\%.

\subsection{General Procedure for Synthesis of Compounds 30-34(Figure 5)}

Compound $20(0.01 \mathrm{~mol})$ was added to an ethanolic sodium ethoxide solution (prepared by 0.23 gm of sodium metal and $30 \mathrm{~mL}$ of ethanol), then ethyl acetoacetate and/or ethyl cyanoacetate $(0.01 \mathrm{~mol})$ was added. The reaction mixture was refluxed for 2hours. After cooling, the mixture was poured into ice/water mixture, then acidified with hydrochloric acid, The product was collected by filtration and washed several times with cold water.

\subsubsection{N-(1,5-dimethyl-3-oxo-2-phenyl-2,3-dihydro-1H- pyrazol-4-yl)-N-((1,5-dimethyl-3-oxo-2-phenyl-2,3-dih ydro-1H-pyrazol-4-yl)carbamothioyl)-3-oxobutanami de (30)}

AcOH; Yield 73\%; white crystals; $m p$ 243-245 ${ }^{\circ}$; IR ( $\left.\mathrm{KBr}\right)$ : $\left(\mathrm{v} / \mathrm{cm}^{-1}\right)=3263(\mathrm{NH}), 3036(\mathrm{CH}$ aromatic $), 2925(\mathrm{CH}$ aliphatic), 1772, 1665 (CO); MS (m/z): $362(\mathrm{M}+, 1.60 \%)$; Anal. Calcd. For $\mathrm{C}_{27} \mathrm{H}_{28} \mathrm{~N}_{6} \mathrm{O}_{4} \mathrm{~S}$ (532): Calcd.: C, 60.90; H, 
5.26; N, 15.78\%; Found: C, 60.85; H, 5.23; N, 15.71\%.

\subsubsection{2-cyano-N-(1,5-dimethyl-3-oxo-2-phenyl-2,3-dihydro- 1H-pyrazol-4-yl)-N-((1,5-dimethyl-3-oxo-2-phenyl-2,3 -dihydro-1H-pyrazol-4-yl)-carbamothioyl)acetamide (32)}

AcOH; Yield $87 \%$; white crystals; mp $227-229^{\circ} \mathrm{C}$; IR ( $\mathrm{KBr}$ ): $\left(v / \mathrm{cm}^{-1}\right)=3263(\mathrm{NH}), 3057(\mathrm{CH}$ aromatic $), 2977(\mathrm{CH}$ aliphatic), 2258(CN), 1687,1642 (CO); MS (m/z): $515(\mathrm{M}+$, 8.26\%).; Anal. Calcd. For $\mathrm{C}_{26} \mathrm{H}_{25} \mathrm{~N}_{6} \mathrm{O}_{3} \mathrm{~S}$ (515): Calcd.: C, 60.58 ; H, 4.85; N, 19.02\%; Found: C, 60.54; H, 4.81; N, $19.00 \%$.

\subsubsection{Methyl 2-(3-(1,5-dimethyl-3-oxo-2-phenyl -2,3-} dihydro-1H-pyrazol-4-yl)-2-((1,5-dimethyl-3-oxo-2-ph enyl-2,3-dihydro-1H-pyrazol-4-yl)amino)-4-oxothiazo lidin-5-ylidene) acetate (34)

A mixture of thiourea $20(0.01 \mathrm{~mol})$ and DMAD $(0.01 \mathrm{~mol})$ in absolute EtOH $(25 \mathrm{~mL})$ was refluxed for 5 hours, then left to cool. The solid product was collected by filtration, washed with ethanol, dried and finally recrystallized from $\mathrm{EtOH}$ yielding thiazolidinone derivative (34) as yellow crystals.

Yield 64\%; mp $140-142^{\circ} \mathrm{C}$; IR $(\mathrm{KBr}):\left(\mathrm{v} / \mathrm{cm}^{-1}\right)=3056(\mathrm{CH}$ aromatic), 2952 (CH aliphatic), 1733,1673 (CO); ${ }^{1} \mathrm{HNMR}$ spectrum, $\delta$, ppm: $2.22,2.54\left(2 \mathrm{~s}, 6 \mathrm{H}, \mathrm{CCH}_{3}\right), 3.17,3.20$ $\left(2 \mathrm{~s}, 6 \mathrm{H}, \mathrm{NCH}_{3}\right), 3.87 \quad\left(\mathrm{~s}, 3 \mathrm{H}, \mathrm{OCH}_{3}\right), 3.93(\mathrm{~s}, 1 \mathrm{H}, \mathrm{C}=\mathrm{CH}), 7.29$ 7.39 (m, $10 \mathrm{H}, \mathrm{Ar}-\mathrm{H})$; Anal. Calcd. For $\mathrm{C}_{28} \mathrm{H}_{26} \mathrm{~N}_{6} \mathrm{O}_{5} \mathrm{~S}$ (558): Calcd.: C, 60.21; H, 4.65; N, 15.05\%; Found: C, 60.18; H, $4.62 ; \mathrm{N}, 15.01 \%$.

\subsection{General Procedure for Synthesis of Compound 39 (Figure 6)}

4-aminoantipyrine (1) $(0.01 \mathrm{~mol})$ was dissolved in $5 \mathrm{ml}$ of dilute $\mathrm{HCl}(1: 1)$. Reaction mass was cooled to $-10^{\circ} \mathrm{C}$ to $-5^{\circ} \mathrm{C}$. Sodium nitrite $(0.7 \mathrm{~g})$ was added in small portions (4 portions) to the reaction mass by maintaining the temperature at $10^{\circ} \mathrm{C}$ to $0^{\circ} \mathrm{C}$ and maintained the reaction for $2 \mathrm{hr}$. A solution of sodium azide $\left(0.7 \mathrm{~g}\right.$ in $\left.20 \mathrm{ml} \mathrm{H}_{2} \mathrm{O}\right)$ was added in a drop wise manner to the reaction mixture at $0^{\circ} \mathrm{C}$. After addition maintained the reaction at $0^{\circ} \mathrm{C}$ for 1 hour.The resulting solid was collected by filtration, washed with water, dried in air overnight after drying for 10 hours, the color of the product change from white to yellow, then recrystallized from methylene chloride .

Yield 68\%; yellow crystals; mp 97-99 ${ }^{\circ} \mathrm{C}$; IR $(\mathrm{KBr}):\left(\mathrm{v} / \mathrm{cm}^{-1}\right)$ $=3065(\mathrm{CH}$ aromatic $), 2984(\mathrm{CH}$ aliphatic $), 1701(\mathrm{CO})$; ${ }^{1} \mathrm{HNMR}$ spectrum, $\delta$, ppm: $3.10\left(\mathrm{~s}, 3 \mathrm{H}, \mathrm{NCH}_{3}\right), 4.03(\mathrm{~s}, 2 \mathrm{H}$, $\left.\mathrm{CH}_{2}\right), 5.29(\mathrm{~s}, 1 \mathrm{H}, \mathrm{CH}), 7.17-7.77(\mathrm{~m}, 5 \mathrm{H}, \mathrm{Ar}-\mathrm{H}) ; \mathrm{MS}(\mathrm{m} / \mathrm{z})$ : 201 (M+, 8.26\%).; Anal. Calcd. For $\mathrm{C}_{11} \mathrm{H}_{11} \mathrm{~N}_{3} \mathrm{O}$ (201): Calcd.: C, 65.67; H, 5.47; N, 20.89\%; Found: C, 65.63; H, 5.42; N, $20.85 \%$.

\subsection{Antimicrobial Activity}

The standardized disc - agar diffusion method [32] was followed to determine the activity of the synthesized compounds against the tested microorganisms.

\subsubsection{Test Organisms}

Cultures of the following microorganism were used in the test: Gram- positive bacteria: Staphylococcus aureus (ATCC 25923) and Bacillus subtilis (ATCC 6635), Gram - negative bacteria: Escherichia coli (ATCC 25922) and Salmonella typhimurium (ATCC 14028), Yeast: Candida albicans (ATCC 10231) and Fungus: Aspergillus fumigatus.

\subsubsection{Screening for the Antimicrobial Potential}

\section{(i) Preparation of Tested Compound}

The tested compounds were dissolved in dimethyl formamide (DMF) solvent and prepared in two concentrations; 100 and $50 \mathrm{mg} / \mathrm{ml}$ and then $10 \mu \mathrm{l}$ of each preparation was dropped on disk of $6 \mathrm{~mm}$ in diameter and the concentrations became 1 and $0.5 \mathrm{mg} /$ disk respectively. In the case of insoluble compounds, the compounds were suspended in DMF and vortexed then processed.

\section{(ii) Testing for anti-Bacterial and Yeasts Activity}

Bacterial cultures were grown in nutrient broth medium at $30^{\circ} \mathrm{C}$. After $16 \mathrm{~h}$ of growth, each microorganism, at a concentration of $10^{8}$ cells $/ \mathrm{mL}$, was inoculated on the surface of Mueller-Hinton agar plates using sterile cotton swab. Subsequently, uniform size filter paper disks $(6 \mathrm{~mm}$ in diameter) were impregnated by equal volume $(10 \mu \mathrm{l})$ from the specific concentration of dissolved compounds and carefully placed on surface of each inoculated plate. The plates were incubated in the upright position at $36^{\circ} \mathrm{C}$ for 24 hours. Three replicates were carried out for each extract against each of the test organism. Simultaneously, addition of the respective solvent instead of dissolved compound was carried out as negative controls. After incubation, the diameters of the growth inhibition zones formed around the disc were measured with transparent ruler in millimeter, averaged and the mean values were tabulated.

\subsubsection{Testing for anti-fungal Activity}

Active inoculum for experiments were prepared by transferring many loopfuls of spores from the stock cultures to test tubes of sterile distilled water (SDW) that were agitated and diluted with sterile distilled water to achieve optical density corresponding to $2.0 \times 10^{5} \mathrm{spore} / \mathrm{ml}$. inoculum of $0.1 \%$ suspension was swabbed uniformly and the inoculum was allowed to dry for 5 minutes then the same procedure was followed as described above.

\subsubsection{Standard References}

The antibiotic chloramphencol was used as standard reference in the case of Gram - negative bacteria, Cephalothin was used as standard reference in the case of Gram - positive bacteria and cycloheximide was used as standard reference in the case of yeasts and fungi.

\subsubsection{Activity Index}

The activity of tested compounds were categorized as follows:

a) Low activity $=$ Mean of zone diameter $\leq 1 / 3$ of mean zone diameter of control. 
b) Intermediate activity $=$ Mean of zone diameter $\leq 2 / 3$ of mean zone diameter of control.

c) High activity $=$ Mean of zone diameter $>2 / 3$ of mean zone diameter of control.

\section{Results and Discussion}

In this investigation, a series of new compounds incorporating rhodanine, thiazole, quinazolin-4-one, imidazolone and pyranothiazole moieties that attached to antipyrine moiety $(3-39)$, was designed and synthesized.
However, treatment of 4-aminoantipyrine with carbon disulfide in ethanol in the presence of potassium hydroxide gave no isolable potassium salt 2. Methyl (1,5-dimethyl-3-oxo -2-phenyl -2,3-dihydro- $1 H$ - pyrazol-4-yl) carbamodithioate (3) and benzyl

(1,5-dimethyl-3-oxo-2-phenyl-2,3-dihydro-1 $H$-pyrazol-4-yl)carbamodithio-ate (4) were prepared from alkylation of the potassium salt 2 with methyl iodide and benzyl chloride, respectively, Figure 1 . The ${ }^{1} \mathrm{H}$ NMR spectra of 3 and 4 revealed the characteristic methyl sulfanyl proton at $\delta=2.60$ $\left(\mathrm{SCH}_{3}\right)$ and $4.53\left(\mathrm{SCH}_{2}\right)$.

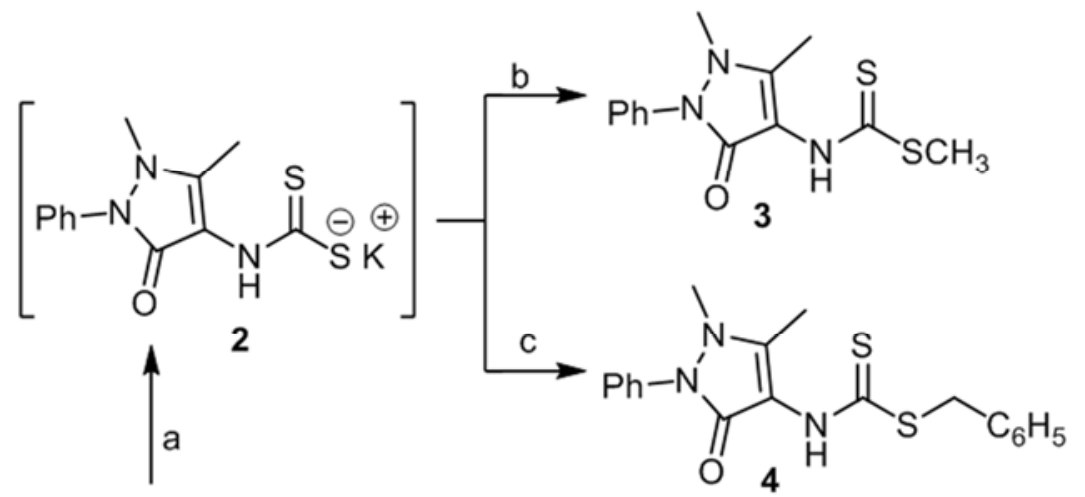<smiles>COC(=O)/C=C1\SC(=S)N(c2c(C)n(C)n(-c3ccccc3)c2=O)C1=O</smiles>

Figure 1. Reagents and conditions: a) $\mathrm{CS}_{2} / \mathrm{KOH} / \mathrm{EtOH}$; b) $\mathrm{CH}_{3} \mathrm{I}$; c) $\mathrm{C}_{6} \mathrm{H}_{5} \mathrm{CH}_{2} \mathrm{Cl}$.

Multi-component reaction (MCR) of 4-aminoantipyrine with carbon disulfide and DMAD was investigated $[17,18]$. Thus, the one-pot reaction of the antipyrine 1 with carbon disulfide and dimethyl acetylenedicarboxylate in ethanol at room temperature under stirring gave rhodanine derivative which identified as methyl 3-(1,5-dimethyl-3-oxo2-phenyl-2,3-dihydro-1 $H$-pyrazol-4-yl)-4-oxo-2-thioxo-1,3-t hiazolidin- 5- ylidene] ethanoate (7) . As shown in Figure 1, the proposed mechanism suggested that the initial addition of the aminoantipyrine to carbon disulfide would result in the formation of the reactive antipyrinyl ammonium carbodithioate 5, which would react with the acetylenic ester of DMAD to yield intermediate 6. Subsequent cyclization of 6 would occur with the loss of methanol to give rhodanine 7 . Reaction of the potassium salt 2 with chloroacetone afforded thiazole derivative, which identified as 1,5-dimethyl-4-(4-
methyl-2-thioxo-1,3-thiazol-3(2H)-yl)-2-phenyl-1,2-dihydro$3 H$-pyrazol-3-one (8), while treatment the potassium salt with sodium chloroacetate then hydrochloric acid give rhodanine derivative, which identified as 3- (1,5-dimethyl-3-oxo2-phenyl-2,3-dihydro-1 $H$-pyrazol-4-yl)-2-thioxo-1,3-thiazoli d-in-4-one (9), Figure 3 .Compound 9 having an active methylene group adjacent to a carbonyl group. Thus, Coupling of 9 with diazonium chloride of 4-aminoantipyrine in ethanolic sodium acetate solution gave azo derivative 10, Figure 3. Enaminone 11 was produce via the reaction of rhodanine 9 with dimethylformamide dimethylacetal in benzene under reflux in good yield. The ${ }^{1} \mathrm{H}$ NMR spectra of 11 revealed the characteristic dimethylamino protons at $\delta 3.20$ $\left(\mathrm{N}\left(\mathrm{CH}_{3}\right)_{2}\right)$. Nitrosation of 9 in acetic acid and sodium nitrite gave nitroso derivative which identified as 3-(1,5-dimethyl3-oxo-2-phenyl-2,3-dihydro-1H-pyrazol-4-yl)-5-nitroso-2-thi 
oxothiazolidin-4-one (12), which present in hydroxyl imino form 13 according ${ }^{1} \mathrm{HNMR}$ spectra which revealed singlet proton at $\delta 14$ of $\mathrm{OH}$ group. Moreover, the reaction of 9 with the aromatic aldehydes namely (p-methoxybenzaldehyde and ethyl vanillin) in ethanol containing a catalytic amount of piperidine afforded the arylidine derivatives 14a,b, respectively, Figure $2 .{ }^{1} \mathrm{H}$ NMR of $14 \mathrm{a}, \mathrm{b}$ showed the presence of a singlet at $\delta 3.86 \mathrm{ppm}$ corresponding to the $\mathrm{OCH}_{3}$ group and triplet at $\delta 1.37$ and quartet at $\delta 4.25 \mathrm{ppm}$ for the $\mathrm{OCH}_{2} \mathrm{CH}_{3}$. reaction of rhodanine 9 with cinnamonitrile $15 \mathrm{a}, \mathrm{b}$ in ethanol in the presence of piperidine as catalyst afforded pyranothiazole derivative $16 \mathrm{a}, \mathrm{b}$, Figure 2 . The IR spectra of compounds $16 \mathrm{a}, \mathrm{b}$ showed the presence of absorption bands at $3385,3330,3386,3324$ and 2220, $2193 \mathrm{~cm}^{-1}$ could be attributed to $\mathrm{NH}_{2}$ and $\mathrm{CN}$ groups, respectively. Further confirmation of the structure of compound 16 was obtained through its synthesis via another reaction route. Thus, the reaction of malononitrile with 14 gave the same product 16 (m.p. and mixed m.p. and finger print IR), Figure 2.<smiles>CCCCCCCCCCCCCCCCn1c(=O)c(-n2c(C)csc2=S)c(C)n1C</smiles>

(2)

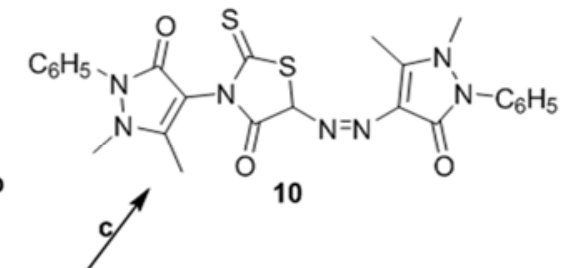

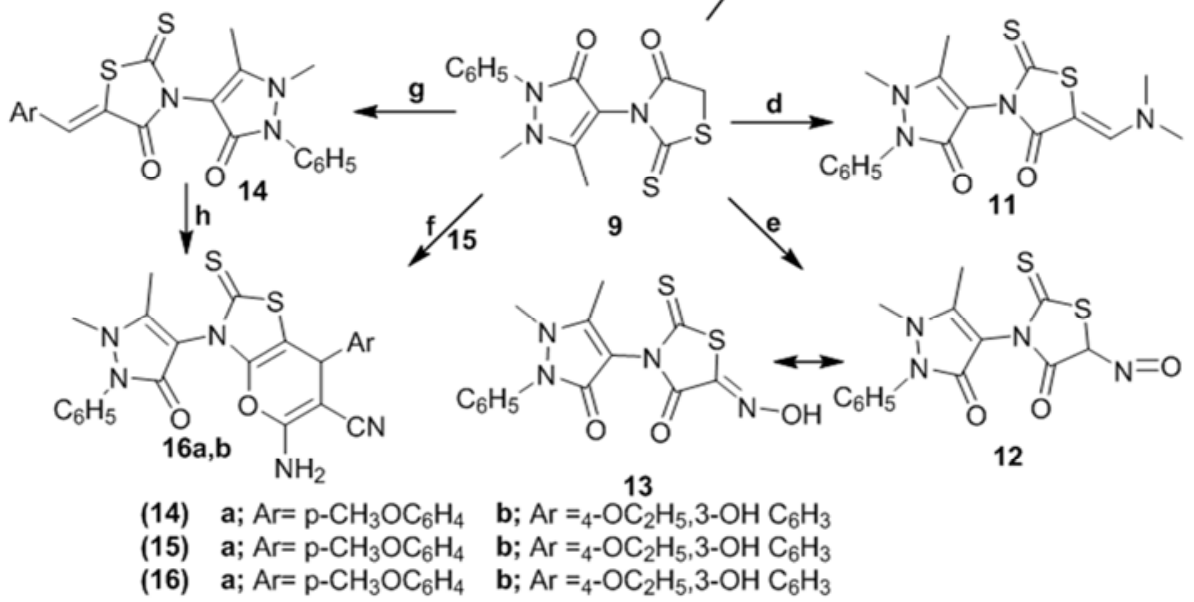

Figure 2. Reagents and Conditions: a) $\mathrm{ClCH}_{2} \mathrm{COCH}_{3}$; b) $\mathrm{ClCH}_{2} \mathrm{COONa} / \mathrm{HCl}$; c) antipyrinedia-zonium chlorided/ EtOH/ AcONa; d) DMFDMA/C $\mathrm{H}_{6}$; e) $\mathrm{NaNO}_{2} / \mathrm{AcOH}$; f) 15; $\mathrm{ArCH}=\mathrm{C}(\mathrm{CN})_{2} / \mathrm{EtOH} / \mathrm{Pip}$.;g) $\mathrm{ArCHO} / \mathrm{EtOH} / \mathrm{Pip}$.; h) $\mathrm{CH} 2(\mathrm{CN})_{2} / \mathrm{EtOH} / \mathrm{Pip}$.

The methylene group present in the rhodanine compound 9 was reported to show high reactivity towards thiazole formation via its reaction with phenylisothiocyanate in basic DMF solution followed by heterocyclization with $\alpha$-halocarbonyl compounds. Thus, 9 was reacted with phenyl isothiocyanate in $\mathrm{DMF} / \mathrm{KOH}$ solution to give the intermediate potassium sulfide salt 17.The reaction of potassium salt with ethyl bromoacetate and chloroacetone afforded thiazolidinone and thiazole derivative 18 and 19, respectively, Figure 3.

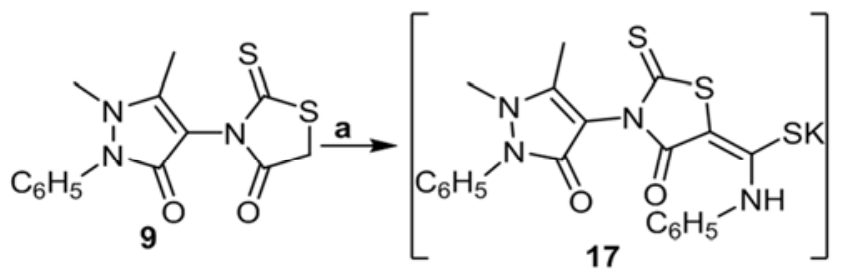

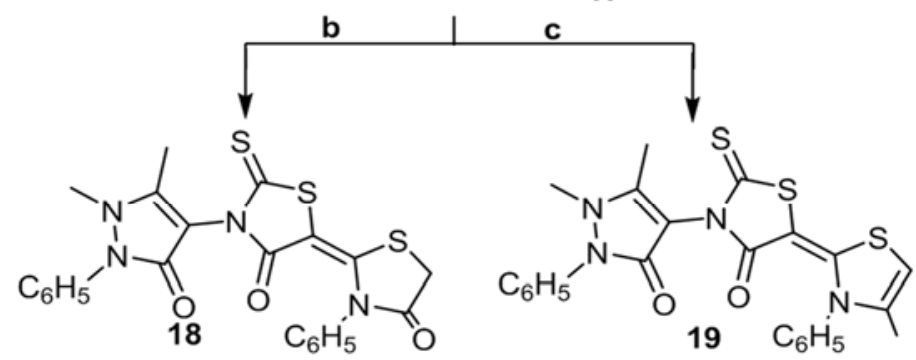

Figure 3. Reagents and Conditions: a) PhNCS/KOH/DMF; b) $\mathrm{BrCH}_{2} \mathrm{COOEt}$; c) $\mathrm{ClCH}_{2} \mathrm{COCH}_{3}$. 
Interaction of compound 3 with hydrazine in hot ethanol furnished pyrazolotriazine 24 instated of thiosemicarbazide derivative 23, Figure 4. The formation of compound 24 indicated by the disappearance absorption band due to $\mathrm{NH}_{2}$ and $\mathrm{CO}$ in its IR spectrum.

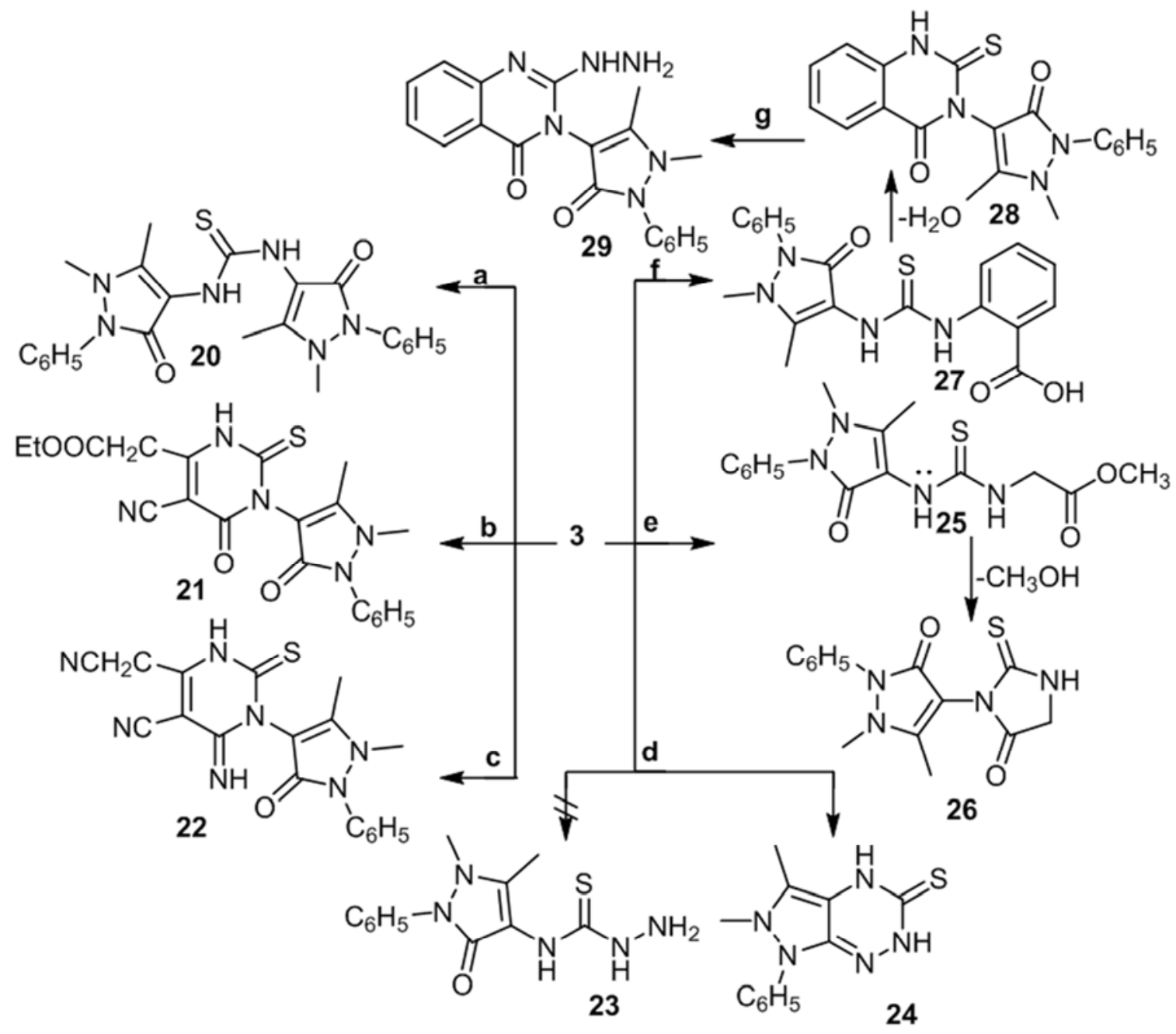

Figure 4. Reagents and Conditions: a)4-aminoantipyrine/EtOH/TEA; b) EtOOC $\left(\mathrm{NH}_{2}\right) \mathrm{C}=\mathrm{C}(\mathrm{CN}) \mathrm{CH}_{2} \mathrm{COOEt} / \mathrm{EtOH} / \mathrm{TEA}$; c) $\mathrm{NC}\left(\mathrm{NH} \mathrm{H}_{2}\right) \mathrm{C}=\mathrm{C}(\mathrm{CN}) \mathrm{CH} 2 \mathrm{CN} /$ EtOH/TEA; d) $\mathrm{NH}_{2} \mathrm{NH}_{2} / \mathrm{EtOH}$; e) GlyOMe.HCl/EtOH/TEA; f); anthranilic acid/EtOH/TEA; g) $\mathrm{NH}_{2} \mathrm{NH}_{2} / \mathrm{EtOH}$.

The molecular ion peak recorded in the mass spectrum was at $\mathrm{m} / \mathrm{z} 259$, which is in agreement with the molecular formula $\left(\mathrm{C}_{12} \mathrm{H}_{13} \mathrm{~N}_{5} \mathrm{~S}\right)$. The investigation was extended to study the reactivity of compound 3 with $\alpha$ - amino acids .Thus, reaction of compound 3 with methyl glycinate hydrochloride in ethanol in the presence of TEA under reflux afforded non isolable thiourea derivative 25 which cyclized to imidazolone derivative which identified as 1,5-dimethyl-4- (5-oxo2-thioxoimidazolidin-1-yl)-2-phenyl-1,2-dihydro-3 $H$-pyrazol -3-one (26) via elimination of methanol molecule, Figure 4. The ${ }^{1} \mathrm{H}$ NMR spectra of 26 revealed the characteristic methylene proton at $\delta 4.34\left(\mathrm{CH}_{2}\right)$. Also, the literature indicated that methyl dithiocarbamate group in compound 3 reacts with anthranilic acid or methyl anthrnilate, leading to the formation of quinazoline and its derivatives [19-23]. Thus, interaction of 3 with anthranilic acid in refluxing ethanol in the presence of TEA as catalyst to yield the thiourea intermediate 27 which underwent cyclization via loss of water molecule yielding quinazoline derivative 28,Figure 4 . When the latter compound 28 was heated in refluxing ethanol with hydrazine hydrate, the hydrazide derivative 29 was obtained via $\mathrm{H}_{2} \mathrm{~S}$ elimination, Figure 4. In the present work the reactivity of thiourea derivative 20 towards bifunctional reagents such as ethyl acetoacete (EAA), ethyl cyanoacetate (ECA) and dimethyl acetylene- dicarboxylate (DMAD) was investigated .Thus, condensation of thiourea 20 with ethyl acetoacetate in ethanolic sodium ethoxide solution gave only one isolable product (TLC) from two proposed structures 30 or 31 . Structure 31 was ruled out on the basis of IR spectrum of the isolated product. Thus, the IR spectrum of (30) revealed absorption bands at 3263 and $1772 \mathrm{~cm}^{-1}$ due to $\mathrm{NH}$ and $\mathrm{CO}$ groups, while its mass spectrum of which was compatible with the molecular formula $\mathrm{C}_{27} \mathrm{H}_{28} \mathrm{~N}_{6} \mathrm{O}_{3} \mathrm{~S}\left(\mathrm{M}^{+}\right.$; 479). Also, under the same experimental conditions thiourea derivative 20 reacted with ethyl cyanoacetate gave open structure 32 instated of pyrimidine derivative 33, Figure 5. The structure 
of pyrimidine derivative 33 was ruled out on the basis of IR spectrum of the isolated product. Thus, the IR spectrum of 32 revealed absorption bands at 3263 and $2258, \mathrm{~cm}^{-1}$ due to $\mathrm{NH}$ and $\mathrm{CN}$ groups, while its mass spectrum of which was compatible with the molecular formula $\mathrm{C}_{26} \mathrm{H}_{25} \mathrm{~N}_{6} \mathrm{O}_{3} \mathrm{~S}\left(\mathrm{M}^{+}\right.$; 515). Heating thiourea derivative 20 with dimethyl acetylene dicarboxylate in ethanol furnished thiazolidinone derivative 34, Figure 5.

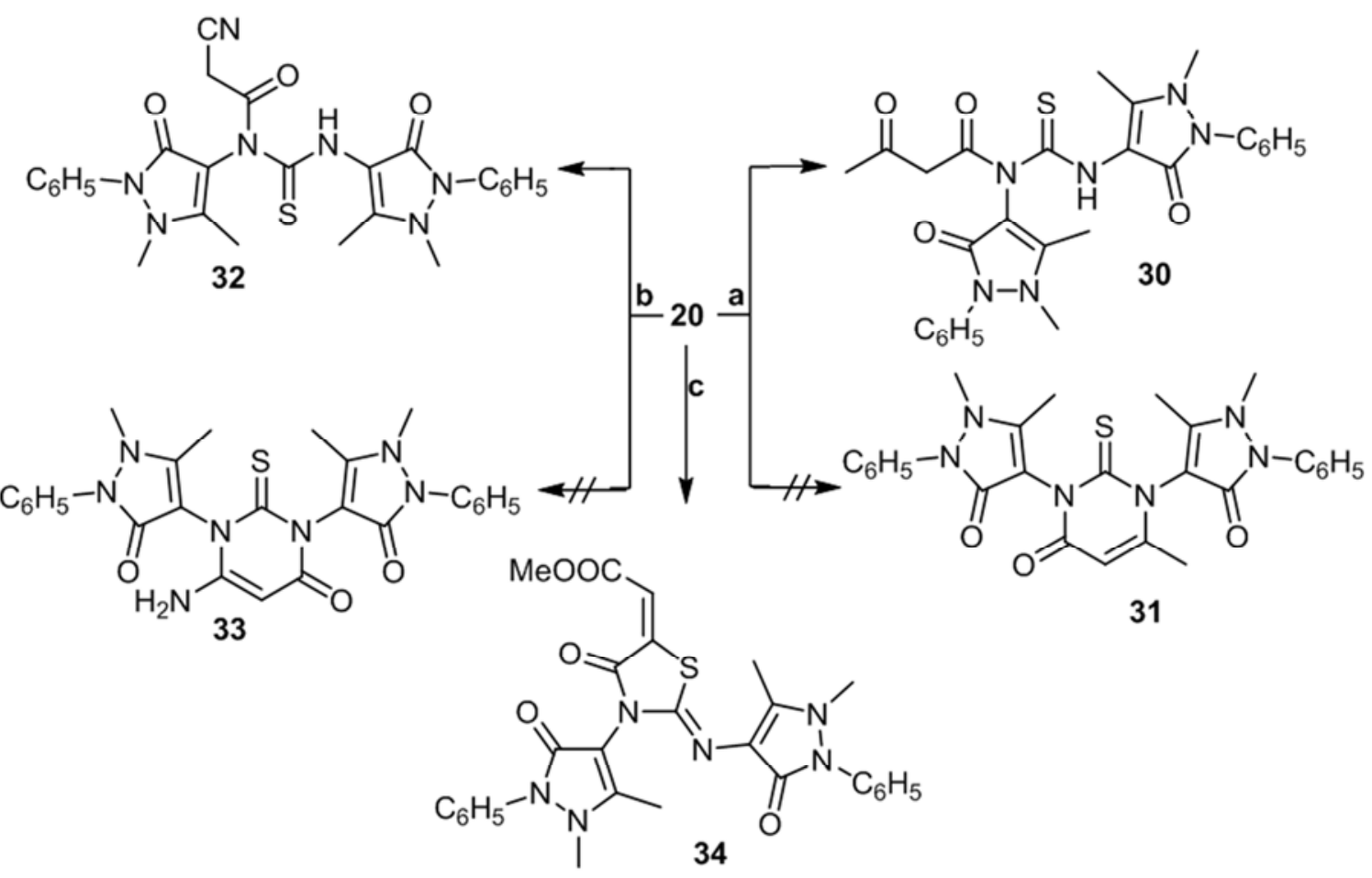

Figure 5. Reagents and Conditions: a) $\mathrm{CH}_{3} \mathrm{COCH}_{2} \mathrm{COOEt} / \mathrm{EtONa}$; b) $\mathrm{CNCH}_{2} \mathrm{COOEt} / \mathrm{EtONa}$; c) DMAD/EtOH.

Finally, the investigation was extended to synthesis of azido antipyrine to use it in the synthesis of 1,2,3-triazole incorporating antipyrine moiety, all attempts failed to obtain this compound (37). In previous publications [24], it has been reported that, isolation of azido antipyrine 36 , then reacted with malononitrile to afford 1,2,3-triazole derivative 37, which cannot be prepared in the present investigation. In the present work, the diazotization of 4-aminoantipyrine (1) by nitrous acid as gave non-isolable antipyrine diazonium chloride 35 . Treatment the latter compound with sodium azide solution, afforded azido antipyrine 36 as white solid, which isolated by filtration, leave this precipitate overnight to dry, the color changed to brown solid. The reaction involved initial formation of azide 36, which loss nitrogen molecule to produce nitrenes intermediate 38 , which undergoes rearrangement to yield the triazine derivative [25-31], which identified as 1-methyl-6-methylidene-2-phenyl -1,6-dihydro -1,2,4-triazin-3(2H)-one (39), Figure 6.

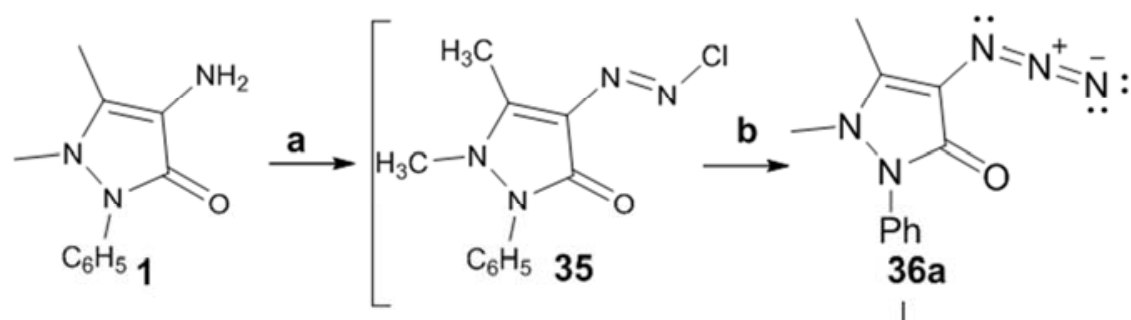

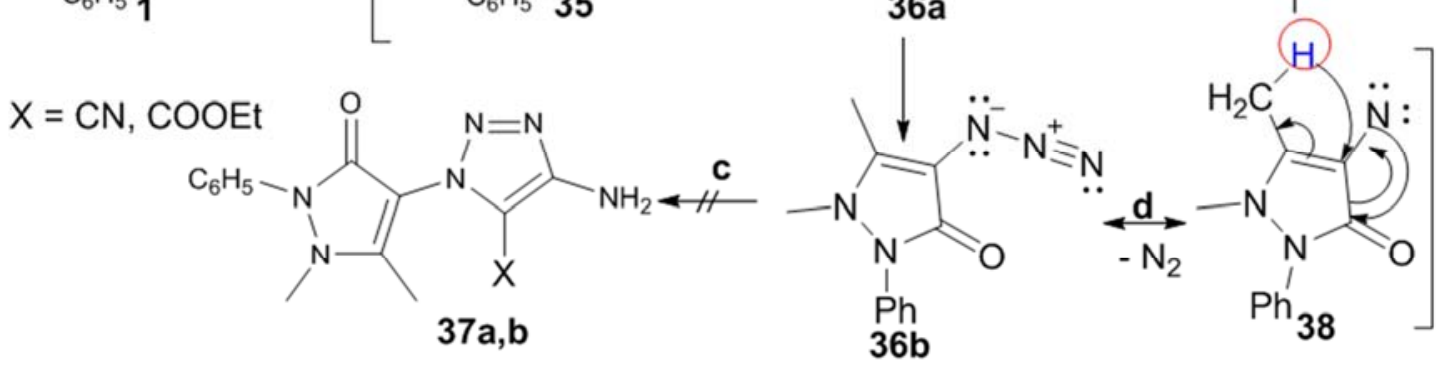

Figure 6. Reagents and Conditions: a) $\mathrm{NaNO}_{2} / \mathrm{HCl}$; b) $\mathrm{NaN}_{3}$ c) $\mathrm{CNCH}_{2} \mathrm{CN}$ and/or $\mathrm{CNCH}_{2} \mathrm{COOEt} / \mathrm{NaOEt}$; d) overnight. 


\section{Antibacterial and Antifungal Activities}

The antibacterial and antifungal activities of the synthetic compounds were assayed against strains of both gram-positive, gram-negative pathogenic bacteria and yeasts and fungi. Initially, the susceptibility testing was carried out by agar diffusion method. The inhibitory effects of the synthetic compounds against these organisms are given in Table 1 . The screening results indicate that: compounds 9, 10 and 18 showed significant antifungal activity with Candida albicans (ATCC10231) and Aspergillus fumigates, while, compounds 7 and 21 showed highest activity against Candida albicans (ATCC10231) due to carboxymethyl group, compound 8 showed highest activity against Aspergillus fumigatus, all the synthetic compounds showed less active against all microorganisms (Gram - positive bacteria, Gram - negative bacteria).

Table 1. The Biological activity for compounds $(3,7,8,9,10,11,13,14 b, 16 b, 18,21,22,26,28$ and 32).

\begin{tabular}{|c|c|c|c|c|c|c|c|c|c|c|c|c|}
\hline \multirow{4}{*}{$\begin{array}{l}\text { Organism } \\
\text { Concentration } \\
\text { Sample } \\
\end{array}$} & \multicolumn{12}{|c|}{ Mean* of zone diameter, nearest whole $\mathrm{mm}$. } \\
\hline & \multicolumn{4}{|c|}{ Gram-positive bacteria } & \multicolumn{4}{|c|}{ Gram-negative bacteria } & \multicolumn{4}{|c|}{ Yeasts and Fungi** } \\
\hline & \multicolumn{2}{|c|}{$\begin{array}{l}\text { Staphylococcus aureus } \\
\text { (ATCC 25923) }\end{array}$} & \multicolumn{2}{|c|}{$\begin{array}{l}\text { Bacillus subtilis } \\
\text { (ATCC 6635) }\end{array}$} & \multicolumn{2}{|c|}{$\begin{array}{l}\text { Salmonella typhimurium } \\
\text { (ATCC 14028) }\end{array}$} & \multicolumn{2}{|c|}{$\begin{array}{l}\text { Escherichia coli } \\
(\text { ATCC 25922) }\end{array}$} & \multicolumn{2}{|c|}{$\begin{array}{l}\text { Candida albicans } \\
\text { (ATCC 10231) }\end{array}$} & \multicolumn{2}{|c|}{$\begin{array}{l}\text { Aspergillus } \\
\text { fumigatus }\end{array}$} \\
\hline & $1 \mathrm{mg} / \mathrm{ml}$ & $0.5 \mathrm{mg} / \mathrm{ml}$ & $\begin{array}{l}\mathbf{m g} / \mathrm{ml} \\
\end{array}$ & $\begin{array}{l}0.5 \\
\mathrm{mg} / \mathrm{ml} \\
\end{array}$ & $1 \mathrm{mg} / \mathrm{ml}$ & $0.5 \mathrm{mg} / \mathrm{ml}$ & $\begin{array}{l}\mathrm{I} \\
\mathrm{mg} / \mathrm{ml}\end{array}$ & $\begin{array}{l}0.5 \\
\mathrm{mg} / \mathrm{ml} \\
\end{array}$ & $1 \mathrm{mg} / \mathrm{ml}$ & $\begin{array}{l}0.5 \\
\mathrm{mg} / \mathrm{ml} \\
\end{array}$ & $\begin{array}{l}\mathrm{I} \\
\mathrm{mg} / \mathrm{ml}\end{array}$ & $\begin{array}{l}0.5 \\
\mathrm{mg} / \mathrm{ml}\end{array}$ \\
\hline 3 & - & - & $9 \mathrm{~L}$ & $8 \mathrm{~L}$ & $10 \mathrm{~L}$ & $7 \mathrm{~L}$ & - & - & $16 \mathrm{I}$ & $13 \mathrm{I}$ & $9 \mathrm{~L}$ & $7 \mathrm{~L}$ \\
\hline 7 & - & - & $16 \mathrm{I}$ & $13 \mathrm{I}$ & - & - & $8 \mathrm{~L}$ & $7 \mathrm{~L}$ & $35 \mathrm{H}$ & $30 \mathrm{H}$ & - & - \\
\hline 9 & $21 \mathrm{I}$ & $16 \mathrm{I}$ & $12 \mathrm{~L}$ & $9 \mathrm{~L}$ & $15 \mathrm{I}$ & $11 \mathrm{I}$ & $19 \mathrm{I}$ & $15 \mathrm{I}$ & $41 \mathrm{H}$ & $36 \mathrm{H}$ & $32 \mathrm{H}$ & $29 \mathrm{H}$ \\
\hline 10 & $16 \mathrm{I}$ & $13 \mathrm{I}$ & $18 \mathrm{I}$ & $15 \mathrm{I}$ & $15 \mathrm{I}$ & $11 \mathrm{I}$ & $18 \mathrm{I}$ & $15 \mathrm{I}$ & $38 \mathrm{H}$ & $34 \mathrm{H}$ & $29 \mathrm{H}$ & $25 \mathrm{H}$ \\
\hline 11 & - & - & $11 \mathrm{~L}$ & $9 \mathrm{~L}$ & - & - & - & - & $20 \mathrm{I}$ & $16 \mathrm{I}$ & - & - \\
\hline 13 & $8 \mathrm{~L}$ & $7 \mathrm{~L}$ & $11 \mathrm{~L}$ & $9 \mathrm{~L}$ & $10 \mathrm{~L}$ & $7 \mathrm{~L}$ & $11 \mathrm{~L}$ & $8 \mathrm{~L}$ & $36 \mathrm{H}$ & $31 \mathrm{H}$ & - & - \\
\hline $14 b$ & - & - & - & - & - & - & - & - & $23 \mathrm{I}$ & $20 \mathrm{I}$ & - & - \\
\hline $16 \mathrm{~b}$ & - & - & - & - & - & - & - & - & 19 & 16 & - & - \\
\hline 18 & - & - & - & - & - & - & - & - & $32 \mathrm{H}$ & $27 \mathrm{H}$ & $33 \mathrm{H}$ & $28 \mathrm{H}$ \\
\hline 22 & - & - & - & - & - & - & - & - & - & - & - & - \\
\hline 26 & $8 \mathrm{~L}$ & $7 \mathrm{~L}$ & $9 \mathrm{~L}$ & $7 \mathrm{~L}$ & - & - & - & - & $16 \mathrm{I}$ & $12 \mathrm{I}$ & - & - \\
\hline 28 & - & - & $9 \mathrm{~L}$ & $8 \mathrm{~L}$ & - & - & $8 \mathrm{~L}$ & $7 \mathrm{~L}$ & - & - & - & - \\
\hline 32 & $11 \mathrm{~L}$ & $9 \mathrm{~L}$ & $8 \mathrm{~L}$ & $7 \mathrm{~L}$ & - & - & $11 \mathrm{~L}$ & $7 \mathrm{~L}$ & $14 \mathrm{I}$ & $9 \mathrm{I}$ & - & - \\
\hline Control \# & 35 & 26 & 35 & 25 & 36 & 28 & 38 & 27 & 35 & 28 & 37 & 26 \\
\hline
\end{tabular}

$*=$ Calculate from 3 values.

** = identified on the basis of routine cultural, morphological and microscopical characteristics.

$-=$ No effect.

L: Low activity $=$ Mean of zone diameter $\leq 1 / 3$ of mean zone diameter of control.

I: Intermediate activity $=$ Mean of zone diameter $\leq 2 / 3$ of mean zone diameter of control.

$\mathrm{H}$ : High activity $=$ Mean of zone diameter $>2 / 3$ of mean zone diameter of control.

\#: Chloramphencol in the case of Gram-positive bacteria, Cephalothin in the case of Gram-negative bacteria and cycloheximide in the case of fungi.

\section{Conclusions}

The new series of heterocyclic derivatives as thiazolidinone, thiazole, imidizalone, qunazolinone and pyranothiazole containing antipyrine moiety were synthesized, using two starting materials, rhodanine 9 and methyl dithiocarbamate 3 derivatives. The antimicrobial properties of some of the prepared compounds were evaluated. The results demonstrate that selected members of this series, including 9, 10 and 18, show excellent activities against all tested Yeast Candida albicans and Fungus Aspergillus fumigates compared with the standard fungicides cycloheximide.

\section{References}

[1] V. C. Filho, R. Correa, Z. Vaz, J. B. Calixto, R. J. Nunes, T. R. Pinheiro, A. D. Andricopulo, R. A. Yunes, Farmaco 53 (1998) 55.

[2] S. M. Sondhi, V. K. Sharma, R. P. Verma, N. Singhal, R.
Shukla, R. Raghubir, M.P. Dubey, Synthesis (1999) 878.

[3] M. M. F. Ismail, Y. A. Ammar, H. S. A. El-Zahaby, S. I. Eisa, S. E. Barakat, Arch. Pharm. Life Sci. 340 (2007) 476.

[4] A. P. Mishra, J. Indian Chem. Soc. 76 (1999) 35.

[5] N. Raman, A. Kulandaisamy, K. Jeyasubramanian, Synth. React. Inorg. Met. 32 (2002) 1583.

[6] N. Raman, A. Kulandaisamy, K. Jeyasubramanian, Synth. React. Inorg. Met. 34 (2004) 17.

[7] S. M. Sondhi, N. Singhal, R. P. Verma, S. K. Arora, S. G. Dastidar, Indian J. Chem., Sect .B, 40 (2001) 113.

[8] M. A. Madiha, A. Rania, H. Moataz, S. Samira, B. Sanaa, Eur. J. Pharmacol. 569(2007) 222.

[9] A. N. Evstropov, V. E. Yavorovskaya, E. S. Vorob, Z. P. Khudonogova, L. N. Gritsenko, E. V. Shmidt, S. G. Medvedeva, V. D. Filimonov, T. P. Prishchep, A. S. Saratikov, Pharm. Chem. J. 26 (1992) 426.

[10] McCrea, J. B.; Vlasses, P.H.; Rocci, M. L., Jr. Ann Pharmacother 23(1989) 38 . 
[11] Meffin, P. J.; Williams, R. L.; Blaschke, T. F.; Rowland, M. J Pharm Sci, 66(1977) 135.

[12] Wensing, G.; Neumann, U.; Ohnhaus, E. E.; Heidemann, H. T. Clini Pharmacother, 48(1990) 575.

[13] M. Yu, M. Kanji, I. Hitoshi, H. Chitoshi, O. Satoru, and S. Takashi, Chemical \& Pharmaceutical Bulletin, vol. 39, no. 6 (1991) 1440-1445.

[14] J. H. Ahn, S. J. Kim, W. S. Park et al., Bioorganic and Medicinal Chemistry Letters, vol. 16, no. 11 (2006) 2996.

[15] M. Sortino, P. Delgado, S. Juarez et al., Bioorganic and Medicinal Chemistry, vol. 15, no. 1 (2007) 484.

[16] T. S. Wan, L. L. Cheng, L. Y. Su, P. L. Siew, and M. S. Mui, Bioorganic \& Medicinal Chemistry Letters, vol. 11 (2001) 91.

[17] Ming-Xia Song, Chang-Ji Zheng, Xian-Qing Deng, Zhi-Yu Wei and Hu-Ri Piao j. medicinal chemistry, 4(5) (2014) 441.

[18] Wael A. A. Arafa, Mervat F. Fareed, Saleh A. Rabeh \& Raafat M. Shaker, phosphorus, sulfur, and silicon, 191(8) (216)1129.

[19] V. Alagarsamy and P. Parthiban J. Heterocyclic Chem., 51, (2014)1615.

[20] M Gobinath1, N Subramanian, V Alagarsamy, S Nivedhitha and V Raja Solomon, Trop J Pharm Res, February; 14(2) (2015)271.

[21] M. Gobinath, N. Subramanian, K. Ruckmani, V. Alagarsamy,
International Journal of Drug Design and Discovery,2(4) (2011)642.

[22] M. Abdalla Hussein, International Journal of Applied Biology and Pharmaceutical Technology, 1(3) (2010)1054.

[23] Hong-Jian Zhang, Peng Jin, Shi-Ben Wang, Fu-Nan Li, Li-Ping Guan, and Zhe-Shan Quan, Arch. Pharm. Chem. Life Sci. 348 (2015) 564.

[24] Aly, H. M.; El-Gazzar, M. G. Arzneimittelforschung, 62 (2012)105.

[25] A. Reiser, H. Wagner, and G. Bowes, Tetrahedron Lett.,7(23) (1966) 2635 .

[26] A. Reiser, G. Bowes, and R. J. Horne, Trans. Faraday SOC., 62 (1966) 3162.

[27] A. Reiser, H. M. Wagner, R. Marley, and G. Bowes, Trans. Faraday SOC, 63 (1967)2403.

[28] L. Horner and A. Christmann, Angew. Chem., 75,707(1963); Angew.Clem. Int.Ed. Engl., 2(1963)599.

[29] G. Labbe Chem. Rev., 69 (3) (1969)345.

[30] P. A. S. Smith, L. 0. Krbechek, AND W. Resemann, Journal of the American Chemical Society, 86 (1964)2025.

[31] N. Svenstrup, K. B. Simonsen, N. Thorup, J. Brodersen, W. Dehaen, and J. Becher, J. Org. Chem., 64 (1999) 2814.

[32] Bauer, A. W., Kirby, W. W. M., Sherris, J. C., and Turck, M., American Journal of Clinical Pathology, 45 (1966) 493. 